\title{
Does Cyclosporine Down Regulate IL-17 in Cardiac Allograft Vasculopathy?
}

\author{
Nandini Nair ${ }^{1}$, Luciano Potena ${ }^{2}$, Sandeep Kumar ${ }^{3}$, Sudhiranjan Gupta ${ }^{3}$, Enrique Gongora ${ }^{4}$, \\ Federica Angeli ${ }^{2}$, Paolo Romani ${ }^{2}$ \\ ${ }^{1}$ Department of Cardiology, Scott \& White Healthcare/Texas A\&M Health Science Center College of Medicine, Temple, USA \\ ${ }^{2}$ Department of Cardiology, University of Bologna, Bologna, Italy \\ ${ }^{3}$ Central Texas VA Health Care System/Texas A\&M Health Science Center College of Medicine, Temple, USA \\ ${ }^{4}$ Division of Cardiothoracic Surgery, Hahnemann University Hospital/Drexel University College of Medicine, Philadelphia, USA \\ Email: nanair@sw.org
}

Received December 11, 2012; revised January 15, 2013; accepted January 23, 2013

\begin{abstract}
Background: Cardiac allograft vasculopathy (CAV) is characterized by vascular inflammation and intimal proliferation which results in luminal stenosis and myocardial infarction. During vascular inflammation elaboration of several cytokines and differential expression of growth factors have been noted. CAV remains the major threat to long-term graft survival. CD4 and CD8 T-cell subsets play a significant role in the development of transplant rejection. Chronic transplant rejection often leads to development of CAV. A new CD4 effector cell subset that produces IL-17 (Th17) has been shown to be up-regulated in the murine system in the setting of CAV. This study assesses the level of IL-17 in cardiac transplant patients with and without CAV as compared to nontransplanted controls. Methods: Levels of IL-17, IL-6, MCP-1 were measured by ELISA in plasma of four nontransplanted controls, nine cardiac allograft recipients with CAV (HT-GVD) and eight post transplant subjects without a diagnosis of CAV (HT-No GVD). All post transplant patients were immune suppressed with cyclosporine. HT-GVD patients were 1 - 15 years post transplant while HT-No GVD subjects were 1 - 10 years post transplant. Results: IL-17, MCP-1 and IL-6 were significantly down regulated in HT-GVD subjects compared to the HT-No GVD subjects $(\mathrm{p}<0.001)$ but not significant between controls and HT-No GVD ( $p=n s)$. Conclusions: A decrease in IL-17 in HT-GVD subjects as compared to HT-No GVD in the presence of cyclosporine treatment could be a consequence of down regulation of IL-6. It is likely that cyclosporine differentially regulates pro inflammatory molecules in the setting of graft vascular disease.
\end{abstract}

Keywords: Allograft; CD4 and CD8 T-Cells; Cardiac Allograft Vasculopathy; IL-17; Luminal Stenosis

\section{Introduction}

Cardiac allograft vasculopathy (CAV) is a complex disease which stems from a range of immunological and nonimmunological insults to the allograft. CAV is characterized by vascular inflammation and intimal proliferation which ultimately results in luminal stenosis of epicardial branches, occlusion of smaller vessels, and myocardial infarction [1-7]. During vascular inflammation elaboration of several cytokines and differential expression of growth factors have been noted. CAV still remains the major threat to long term graft survival. CD4 and CD8 Tcell subsets play an important role in the development of transplant rejection [6]. A new CD4 effector cell subset that produces IL-17 (Th17) has emerged in importance. Th17 cells promote autoimmunity in mice and have been implicated in the pathogenesis of human inflammatory

*This study is limited by the small sample size and should be validated in larger number of subjects. diseases. IL-17 is a pro-inflammatory cytokine that activates $\mathrm{T}$-cells and ultimately results in the production of a variety of other cytokines, chemokines, and cell adhesion molecules. IL-17 has been shown to be up regulated in the murine system in the setting of CAV [7] hence, this study was undertaken to assess the level of IL-17 in CAV as compared to human cardiac allograft recipients without CAV as well as nontransplanted subjects.

\section{Materials and Methods}

Patient samples: Blood was obtained as per IRB approved protocols at Scott \& White Hospital, Temple, TX (four nontransplanted controls) and University of Bologna, Italy (nine transplant patients with CAV (HT-GVD) and eight post-transplant subjects without diagnosis of CAV (HT-No GVD). All post transplant patients were immunesuppressed with cyclosporine. The levels of IL17, IL-6, MCP-1 were measured by ELISA in plasma of 
four nontransplanted controls, seven CAV (HT-GVD), and seven CAV (HT-No GVD) patients. The CAV positive patients ranged from ages 56 - 61 years and CAV negative subjects ranged from 26 - 61 years (Table 1).

Cytokine ELISA: The level of human IL-17 in serum samples were measured using a Single Analyte ELISArray kit (SABiosciences, Frederick, MD) following the manufacturer's instructions. The Single-Analyte ELISArray Kits are designed to quantitatively measure the amount of an individual protein analyte using a standard sandwich enzyme-linked immunosorbent assay (ELISA) technique. A target-specific capture antibody has been coated onto eight well strips placed into a 96-well plate format. Serum samples were added to the wells and incubated at room temperature for three hours, after which the biotinylated secondary antibody was added and incubated for one hour at room temperature. After washing with wash buffer, complementary HRP-conjugated Ab was added and incubated for 30 minutes at room temperature. The plates were washed and developed with the substrate and the reaction was stopped with stop buffer and the OD was read at $450 \mathrm{~nm}$ on a microplate reader (Spectramax 250 microplate reader Molecular Devices, Sunnyvale, CA). The ELISA sensitivity for all the three cytokines assayed IL 6, IL 17 and MCP1 was $25 \mathrm{pg} / \mathrm{mL}$. All analyses were carried out as per manufacture's protocol.

Data interpretation and statistical analyses: The changes observed in the cytokine levels were expressed as $\mathrm{pg} / \mathrm{mL}$. All experiments were performed in triplicate for each determination. Data are expressed as means \pm standard error and analyzed using the Kruskal-Wallis one-way analysis of variance for significance with post tests using Prism 5.0 GraphPad software (GraphPad, San Diego, CA, USA). A p value less than 0.05 was considered statistically significant.

\section{Results}

IL-17 and MCP-1 (Figures 1 and 2) are significantly down regulated in HT-GVD subjects compared to the control subjects $(\mathrm{p}<0.001)$. IL-6 did not show any statistically significant changes between controls and HT-GVD (p = ns) (Figure 3). IL-17 and MCP-1 did not show any changes that were statistically significant between controls and HT-No GVD ( $\mathrm{p}=\mathrm{ns})$. IL-17, MCP-1 and IL-6 were significantly down regulated in HT-GVD subjects compared to the HT-No GVD subjects ( $\mathrm{p}<0.001$ ) but not significant between controls and HTNo GVD $(\mathrm{p}=\mathrm{ns})$. HT-GVD patients were $1-15$ years post transplant while HT-No GVD subjects were 1 - 10 years post transplant.

Table 1. Baseline characteristics of cardiac transplant patients.

\begin{tabular}{|c|c|c|c|c|}
\hline Gender & Age & Years after transplant & Cardiac allograft vasculopathy & Reason for heart transplant \\
\hline M & 61 & 1 & Positive & Ischemic Cardiomyopathy \\
\hline $\mathrm{F}$ & 56 & 2 & Positive & Non ischemic Cardiomyopathy \\
\hline M & 71 & 10 & Positive & Non ischemic Cardiomyopathy \\
\hline M & 63 & 1 & Positive & Ischemic Cardiomyopathy \\
\hline M & 61 & 10 & Positive & Non ischemic Cardiomyopathy \\
\hline $\mathrm{F}$ & 57 & 1 & Positive & Non ischemic Cardiomyopathy \\
\hline M & 61 & 15 & Positive & Non ischemic Cardiomyopathy \\
\hline M & 61 & 15 & Positive & Non ischemic Cardiomyopathy \\
\hline M & 61 & 15 & Positive & Non ischemic Cardiomyopathy \\
\hline $\mathrm{F}$ & 33 & 1 & Negative & Non ischemic Cardiomyopathy \\
\hline M & 34 & 10 & Negative & Non ischemic Cardiomyopathy \\
\hline M & 33 & 5 & Negative & Non ischemic Cardiomyopathy \\
\hline M & 50 & 5 & Negative & Non ischemic Cardiomyopathy \\
\hline M & 32 & 1 & Negative & Non ischemic Cardiomyopathy \\
\hline M & 61 & 5 & Negative & Ischemic Cardiomyopathy \\
\hline $\mathrm{F}$ & 54 & 1 & Negative & Non ischemic Cardiomyopathy \\
\hline M & 26 & 1 & Negative & Non ischemic Cardiomyopathy \\
\hline
\end{tabular}




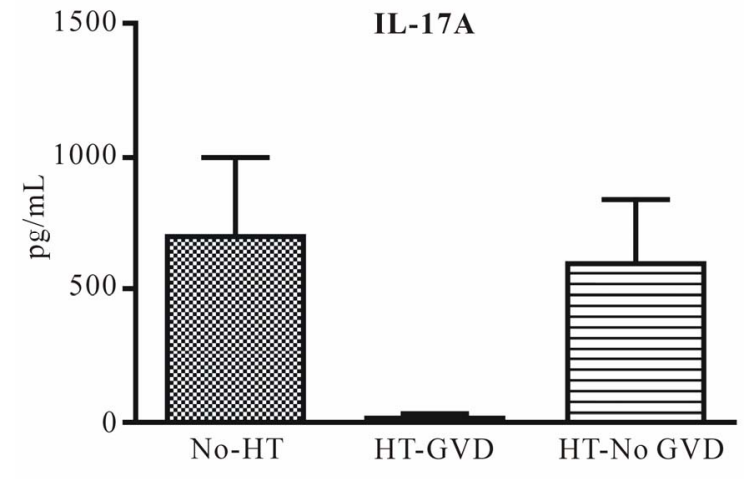

No-HT $=$ non transplanted patients $(n=4)$ HTGVD = patients with diagnosis of CAV $(n=9)$ HT-No GVD = patients without diagnosis of CAV $(n=8)$

Figure 1. IL-17A expression in CAV versus control nontransplanted patients.

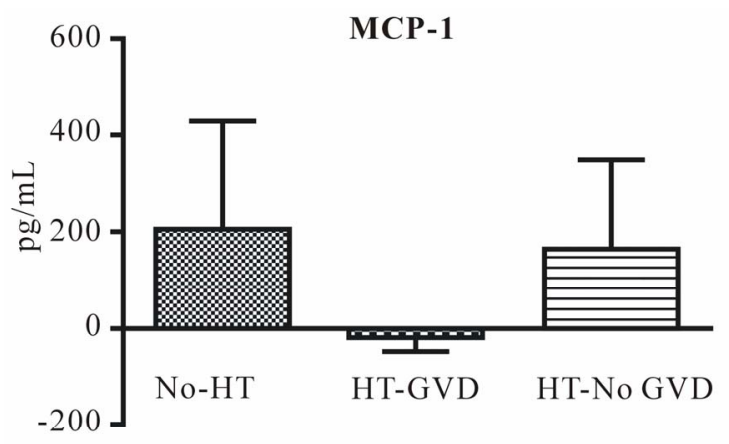

No-HT $=$ non transplanted patients $(n=4)$

HTGVD = patients with diagnosis of CAV $(n=9)$

HT-No GVD = patients without diagnosis of CAV $(n=8)$

Figure 2. MCP-1 expression in CAV versus control non transplanted patients.

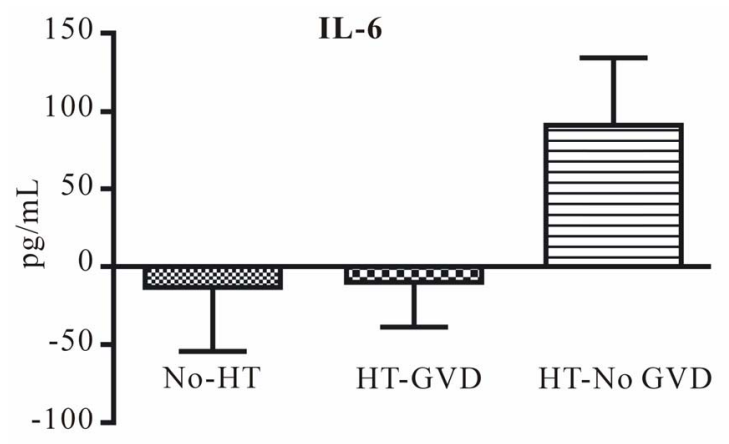

No-HT $=$ non transplanted patients $(n=4)$

HTGVD = patients with diagnosis of CAV $(n=9)$

HT-No GVD = patients without diagnosis of CAV $(n=8)$

Figure 3. IL-6 expression in CAV versus control non transplanted patients.

\section{Discussion}

IL-17 plays a critical role in the pathogenesis of autoimmune and chronic inflammatory disorders and stimulates the induction of various pro inflammatory cytokines and chemokines. In a murine model of chronic allograft vasculopathy, IL-17 mediates an aggressive proinflammatory response culminating in severe accelerated allograft rejection and vasculopathy [7]. A variety of inflammatory and autoimmune diseases such as rheumatoid arthritis, multiple sclerosis, Crohn's disease, psoriasis, Behcet's disease and uveitis, show IL-17 overexpression and production [8-15]. Cyclosporine is known to inhibit production of several inflammatory cytokines, such as IL-17, IL-12, IL-18, and tumor necrosis factor- $\alpha[16,17]$. Several reports have shown that CsA could inhibit IL-17 production in certain autoimmune diseases, such as VogtKoyanagi-Harada (VKH) syndrome [18-20]. It is possible that CsA exerts its function via inhibiting IL-17 and interferon gamma production in Behcet's disease [20]. Other possible pathways include the up regulation of CD69 T-cell-surface activation marker via IL-15 and a calcium ionophore which are also noted in Behcet's disease. In rheumatoid arthritis increased IL17 levels were significantly inhibited at the transcription and translational levels by cyclosporine. This effect was explained by the fact that cyclosporine inhibited the IL-17 production by Th17 cells at the protein and at the mRNA levels. Besides, cyclosporine also markedly reduced the expression of CD69 and CD 25. Therefore cyclosporine may exert its effect through suppression of IL17 production and inhibition of TH17 cell differentiation [21].

The statistically significant down regulation of these proinflammatory cytokines in chronic CAV (HT-GVD) is interesting in the setting of calcineurin inhibition by cyclosporine. The down regulation of IL-17 in patients with CAV (HT-GVD) may reflect the Treg/Th17 balance as imbalance of Th17/Treg has been considered critical in the development of inflammation. IL-6 has a very important role in regulating the balance between IL-17 producing Th17 cells and regulatory T-cells (Treg). Th17 is involved in the pathogenesis of autoimmune responses, while Treg functions to restrain excessive effector T-cell responses. IL-6 induces the development of Th17 cells from naïve T-cells together with TGF-beta and inhibits TGF-beta-induced Treg differentiation. Thus a decrease in IL-17 in HT- GVD subjects as compared to HT- no GVD in the presence of cyclosporine treatment could be a consequence of down regulation of IL-6. It is likely that cyclosporine differentially regulates proinflammatory molecules in the setting of graft vascular disease (GVD). The decrease in MCP-1 as well as IL-6 and IL-17 differentially in the GVD patients is also suggestive of interaction of downstream effects resulting from down regulation of the individual cytokines.

\section{Acknowledgements}

This study was funded by an internal grant from the Di- 
vision of Cardiology to Nandini Nair MD, PhD and Texas A\&M startup funds to Dr Sudhiranjan Gupta. Texas VA Health Science provided the research facility.

\section{REFERENCES}

[1] H. Valantine, "Cardiac Allograft Vasculopathy after Heart Transplantation: Risk Factors and Management," Journal of Heart Lung Transplantation, Vol. 23, No. 5, 2004, pp. S187-S193. doi:10.1016/j.healun.2004.03.009

[2] M. R. Costanzo, D. C. Naftel, M. R. Pritzker, J. K. Heilman, J. P. Boehmer, S. C. Brozena, et al., "Heart Transplant Coronary Artery Disease Detected by Coronary Angiography: A Multi-Institutional Study of Preoperative Donor and Recipient Risk Factors," Journal of Heart and Lung Transplantation, Vol. 17, No. 8, 1998, pp. 744-753.

[3] M. E. Billingham, "Histopathology of Graft Coronary Disease," Journal of Heart and Lung Transplantation, Vol. 11, No. 3, 1992, pp. S38-S44.

[4] F. Moien-Afshari, B. M. McManus and I. Laher, "Immunosuppression and Transplant Vascular Disease: Benefits and Adverse Effects," Pharmacology and Therapeutics, Vol. 100, No. 2, 2003, pp. 141-156. doi:10.1016/j.pharmthera.2003.08.002

[5] S. Radio, S. Wood, J. Wilson, H. Lin, G. Winters and B. McManus, "Allograft Vascular Disease: Comparison of Heart and Other Grafted Organs,” Transplantation Proceedings, Vol. 28, No. 1, 1996, pp. 496-499.

[6] M. Weis, "Cardiac Allograft Vasculopathy: Prevention and Treatment Options," Transplantation Proceeding, Vol. 34, No. 5, 2002, pp. 1847-1849. doi:10.1016/S0041-1345(02)03101-9

[7] X. Yuan, J. Paez-Cortez, I. Schmitt-Knosalla, F. D’Addio, B. Mfarrej, M. Donnarumma, et al., "A Novel Role of CD4 TH17 Cells in Mediating Cardio Allograft Rejection and Vasculopathy," Journal of Experimental Medicine, Vol. 205, No. 13, 2008, pp. 3133-3144. doi:10.1084/jem.20081937

[8] P. Hillyer, M. J. Larché, E. P. Bowman, T. K. McClanahan, R. de W. Malefyt, L. P. Schewitz, et al., "Investigating the Role of the Interleukin-23/-17A Axis in Rheumatoid Arthritis," Rheumatology, Vol. 48, No. 12, 2009, pp. 1581-1589. doi:10.1093/rheumatology/kep293

[9] V. Brucklacher-Waldert, K. Stuerner, M. Kolster, J. Wolthausen and E. Tolosa, "Phenotypical and Functional Characterization of T Helper 17 Cells in Multiple Sclerosis,” Brain, Vol. 132 No. 12, 2009, pp. 3329-3341. doi:10.1093/brain/awp289

[10] Z. J. Liu, P. K. Yadav, J. L. Su, J. S. Wang and K. Fei, "Potential Role of Th17 Cells in the Pathogenesis of Inflammatory Bowel Disease," World Journal of Gastroenterology, Vol. 15, No. 46, 2009, pp. 5784-5788. doi:10.3748/wjg. 15.5784

[11] A. Di Cesare, P. Di Meglio and F. O. Nestle, "The IL23/Th17 Axis in the Immunopathogenesis of Psoriasis," The Journal of Investigatiave Dermatology, Vol. 129, No. 6, 2009, pp. 1339-13350. doi:10.1038/jid.2009.59
[12] W. Chi, P. Yang, B. Li, C. Wu, H. Jin, X. Zhu, et al., "Interleukin-23 Promotes CD4+ T Cells to Produce Interleukin-17 in Vogt-Koyanagi-Harada Disease” The Journal of Allergy and Clinical Immunology, Vol. 119, No. 5, 2007, pp. 1218-1224. doi:10.1016/j.jaci.2007.01.010

[13] W. Chi, X. Zhu, P. Yang, X. Liu, X. Lin, H. Zhou, et al., "Upregulated IL-23 and IL-17 in Behcet Patients with Active Uveitis," Investigative Ophthalmology and Visual Science, Vol. 49, No. 7, 2008, pp. 3058-3064. doi:10.1167/iovs.07-1390

[14] P. Yang, W. Fang, Q. Meng, Y. Ren, L. Xing and A. Kijlstra, "Clinical Features of Chinese Patients with Behcet's Disease,” Ophthalmology, Vol. 115, No. 2, 2008, pp. 312-318. doi:10.1016/j.ophtha.2007.04.056

[15] M. L. Cho, W. U. Kim, S. Y. Min, D. J. Min, J. K. Min, S. H. Lee, et al., "Cyclosporine Differentially Regulates Interleukin-10, Interleukin-15, and Tumor Necrosis Factor Aproduction by Rheumatoid Synoviocytes," Arthritis and Rheumatism, Vol. 46, No. 1, 2002, pp. 42-51. doi:10.1002/1529-0131(200201)46:1<42::AID-ART1002 6>3.0.CO;2-A

[16] R. Giacomelli, P. Cipriani, M. M. Cerinic, A. Fulminis, G. Barattelli, E. Pingiotti, et al., "Combination Therapy with Cyclosporine and Methotrexate in Patients with Early Rheumatoid Arthritis Soon Inhibits TNFalpha Production without Decreasing TNFalpha mRNA Levels: An in Vivo and in Vitro Study," Clinical and Experimental Rheumatology, Vol. 20, No. 3, 2002, pp. 365-372.

[17] X. Liu, P. Yang, X. Lin, X. Ren, H. Zhou, X. Huang, et al., "Inhibitory Effect of Cyclosporin A and Corticosteroids on the Production of IFN- $\gamma$ and IL-17 by T Cells in Vogt-Koyanagi-Harada Syndrome," Clinical Immunology, Vol. 131, No. 2, 2009, pp, 333-342. doi: 10.1016/j.clim.2008.12.007

[18] M. Ziolkowska, A. Koc, G. Luszczykiewicz, K. Ksiezopolska-Pietrzak, E. Klimczak, H. Chwalinska-Sadowska et al., "High Levels of IL-17 in Rheumatoid Arthritis Patients: IL-15 Triggers in Vitro IL-17 Production via Cyclosporin A-Sensitive Mechanism," Journal of Immunology, Vol. 164, No. 5, 2000, pp. 2832-2838.

[19] A. S. Haider, M. A. Lowes, M. Suárez-Fariñas, L. C. Zaba, I. Cardinale, A. Khatcherian, et al., "Identification of Cellular Pathways of 'Type 1', Th17 T Cells, and TNFand Inducible Nitric Oxide Synthase-Producing Dendritic Cells in Autoimmune Inflammation through Pharmacogenomic Study of Cyclosporine A in Psoriasis,” Journal of Immunology, Vol. 180, No. 3, 2008, pp. 1913-1920.

[20] W. Chi, P. Yang, X. Zhu, Y. Wang, L. Chen, X. Huang, et al., "Production of Interleukin-17 in Behcet's Disease Is Inhibited by Cyclosporin A,” Molecular Vision, Vol. 16, 2010, pp. 880-886. http://www.molvis.org/molvis/v16/a98/

[21] C. Zhang, J. Zhang, B. Yang and C. Wu, "Cyclosporin A Inhibits the Production of Il-17 by Memory Th17 Cells from Healthy Individuals and Patients with Rheumatoid Arthritis," Cytokine, Vol. 42, No. 3, 2008, pp. 345-352. doi:10.1016/j.cyto.2008.03.006 